\title{
Histopathological examination of nerve samples from pure neural leprosy patients: obtaining maximum information to improve diagnostic efficiency
}

\author{
Sérgio Luiz Gomes Antunes ${ }^{1 /+}$, Leila Chimelli², Márcia Rodrigues Jardim¹, \\ Robson Teixeira Vital', José Augusto da Costa Nery', Suzana Corte-Real' ${ }^{3}$, \\ Mariana Andréa Vilas Boas Hacker ${ }^{1}$, Euzenir Nunes Sarno ${ }^{1}$
}

\begin{abstract}
'Laboratório de Hanseníase ${ }^{3}$ Laboratório de Biologia Estrutural, Instituto Oswaldo Cruz-Fiocruz, Av. Brasil 4365, 21045-900 Rio de Janeiro, RJ, Brasil ²Departamento de Patologia, Universidade Federal do Rio de Janeiro, Rio de Janeiro, RJ, Brasil
\end{abstract}

Nerve biopsy examination is an important auxiliary procedure for diagnosing pure neural leprosy (PNL). When acid-fast bacilli (AFB) are not detected in the nerve sample, the value of other nonspecific histological alterations should be considered along with pertinent clinical, electroneuromyographical and laboratory data (the detection of Mycobacterium leprae DNA with polymerase chain reaction and the detection of serum anti-phenolic glycolipid 1 antibodies) to support a possible or probable PNL diagnosis. Three hundred forty nerve samples [144 from PNL patients and 196 from patients with non-leprosy peripheral neuropathies (NLN)] were examined. Both AFB-negative and AFB-positive PNL samples had more frequent histopathological alterations (epithelioid granulomas, mononuclear infiltrates, fibrosis, perineurial and subperineurial oedema and decreased numbers of myelinated fibres) than the NLN group. Multivariate analysis revealed that independently, mononuclear infiltrate and perineurial fibrosis were more common in the PNL group and were able to correctly classify AFB-negative PNL samples. These results indicate that even in the absence of $A F B$, these histopathological nerve alterations may justify a PNL diagnosis when observed in conjunction with pertinent clinical, epidemiological and laboratory data.

Key words: leprosy - pure neural form - diagnosis

Leprosy-related peripheral neuropathy is responsible for the disabilities and deformities closely associated with the disease. Despite stringent control measures established by the WHO and a consequent decrease in the disease's worldwide prevalence, the rate of newly detected leprosy cases remains epidemiologically high in a number of countries (WHO 2010).

In addition to the involvement of the skin and peripheral nervous system, leprosy patients may present with peripheral neuropathy (simple or multiple mononeuropathies and/or polyneuropathy) in the absence of any verifiable cutaneous lesions (Pannikar et al. 1983, Girdhar 1996), a state commonly referred to as pure neural leprosy (PNL). The confirmation of a PNL diagnosis requires the demonstrated presence of Mycobacterium leprae in a biopsy of any affected sensory nerve.

Disease morbidity is a consequence of inflammatory infiltrate which is composed of mononuclear cells (macrophages and lymphocytes), epithelioid cells or $M$. leprae-loaded foamy macrophages (Shetty \& Antia 1997) in the nerves, a response to the presence of $M$. leprae. Classical descriptions of histopathological alterations in leprosy-affected nerves have been detailed in pioneering

Financial support: IOC-FIOCRUZ

+ Corresponding author: santunes@ioc.fiocruz.br

Received 19 June 2011

Accepted 1 December 2011 reports that explored the structural nerve damage inherent in this disease (Dastur et al. 1970, Job 1989, Srinivasan 1990, Shetty \& Antia 1997).

Chimelli et al. (1997) described the value of nerve biopsies in diagnosing PNL. They described the most frequent morphological alterations found in nerve samples and underscored the significance of nerve biopsies in differentially diagnosing other neuropathies and as a posttreatment monitoring tool. A similar report published by Garbino et al. (2004) described the important role of nerve biopsies and the immunohistochemical labelling of $M$. leprae antigens in nerve sections in confirming diagnoses. Furthermore, Jardim et al. (2003) established general criteria for determining PNL by interpreting clinical, electroneuromyographical, laboratory and histopathological data from a nerve sample biopsy.

It is worth noting that the value of nerve biopsy examination increases when the results are interpreted in the context of pertinent clinical, epidemiological, electroneuromyographical and laboratory data [i.e., M. leprae DNA determined with polymerase chain reaction (PCR) (Jardim et al. 2003) and the presence of serum antiphenolic glycolipid antibodies (Jardim et al. 2005)]. From this point of view, the final diagnostic decision is the direct responsibility of the clinical assistant, who must consider all the available data. However, nerves do not always contain AFB; they may only show the morphological alterations that developed during the disease's progression, posing an additional challenge for the assistant who must evaluate the remaining data and arrive at the correct diagnosis. 
It is clear that an unequivocal leprosy diagnosis cannot be made with acid-fast bacilli (AFB)-negative sample. Under such circumstances, nerve biopsies have only a relative diagnostic value and the maximum amount of information must be sought from the histological examination of nerves to arrive at a probable diagnosis.

The present paper consists of reflections based on 13 years of experience (1997-2010) with the histopathological analysis of biopsied nerve samples from patients with peripheral neuropathy and suspected PNL. The patients were seen at the Souza Araújo (ASA) Outpatient Service, Leprosy Laboratory, a reference centre accredited by the Brazilian Ministry of Health, in Rio de Janeiro. For AFBnegative samples, the diagnoses were strengthened by clinical, electroneurophysiological and laboratory investigations combined with nerve sample histopathology.

The frequency of histopathological changes in the PNL group was compared with those found in samples from a non-leprosy peripheral neuropathy (NLN) group. In addition, the frequency of histopathological alterations in the AFB-negative PNL samples was compared with the frequency in the AFB-positive PNL and NLN samples. The present study attempted to ascertain the value of each histological alteration in the AFB-negative samples from the PNL group and how such alterations might influence the determination of a PNL diagnosis.

\section{PATIENTS, MATERIALS AND METHODS}

Between 1997-2010, histological biopsy specimens from all 144 PNL and 196 NLN patients attending at ASA were examined and their clinical, epidemiological, nerve conduction studies and laboratory data were subsequently collected. The criteria adopted to diagnose PNL in the present study were proposed by Jardim et al. (2003) and are as follows: the presence of mononeuropathy multiplex or simplex, which may evolve to confluent polyneuropathy with an initially predominant sensory impairment accompanied by more or lessfrequent motor involvement. Autonomic dysfunction, represented by erythrocyanosis, nerve enlargement and nerve pain elicited by palpation are additional signs of leprosy neuropathy. The nerve conduction study may detect a demyelinating pattern represented by increased latency, decreased conduction velocity, conduction block and temporal dispersion. Decreased nerve action potential amplitude can also be a sign of axonal damage. Mixed patterns (axonal plus demyelinating) can also be seen in this exam. Nerve biopsy is important for confirming a leprosy diagnosis, particularly when the biopsy detects an inflammatory infiltrate with the presence of AFB or an epithelioid granuloma. Non-specific nerve alterations, such as fibrosis, endoneurial oedema and perineurial enlargement, make the diagnosis probable. Histopathological findings in line with leprosy in biopsied nerve samples, the detection of $M$. leprae DNA in the nerve with PCR and/or the presence of elevated levels of serum antiphenolic glycolipid antibodies with enzyme linked immunosorbent assay (Jardim et al. 2005) would make a leprosy diagnosis certain, probable or possible. Positive results for any of these laboratory tests are useful for diagnosis only in the context of high- ly suggestive clinical and electroneurophysiological peripheral neuropathy in an endemic area. A diagnosis should never be based on one item alone, particularly in AFB-negative nerve samples (Table I).

The NLN tag was assigned to samples whose total test results were inconsistent with PNL. Aetiological diagnoses for 69 of the 196 NLN patients were determined in ASA after other clinical and laboratory data were analysed. The causes of their neuropathy were as follows: 28 cases were highly suggestive of nerve entrapment, four had diabetes, two had alcohol toxicity, seven had vasculitis, one had post-trauma neuropathy, one had lupus erythematosus, seven had amyloidosis, seven had chronic inflammatory demyelinating neuropathy, one had paraneoplastic syndrome, three had genetic neuropathies (1 Charcot Marie Tooth and 1 peripheral myelin protein duplication), two had multifocal acquired demyelinating motor neuropathy, one had syringomyelia, one had neuropathy of Chagas disease and one had chronic axonal idiopathic polyneuropathy. Although 28 patients had a clinical picture highly suggestive of nerve entrapment, this diagnosis could not be confirmed with imaging techniques. Once it was determined that the aetiology of the peripheral neuropathy that brought the patient to the medical service could not be confirmed, the remaining 127 NLN patients were referred to special clinics for peripheral neuropathy.

The nerves selected for biopsy were the dorsal cutaneous branches of the ulnar (173), sural (107), superficial fibular (22), radial (1) and median nerves (3). Forty of the biopsied nerve samples could not be identified.

The nerve samples were divided into two parts. One was fixed in 4\% paraformaldehyde, dehydrated, clarified with xylene and embedded in paraffin for routine histopathological examination. The paraffin blocks were sectioned into $5-\mu \mathrm{m}$-thick sections with a microtome (Shandon, USA). The sections were laid on glass slides and deparaffinised. They were then rehydrated and stained with haematoxylin-eosin to evaluate inflammatory infiltrate and cellularity with Gomori's trichrome to assess fibrosis and nerve structure and with Wade staining to detect AFB. The other part of the nerve sample was fixed in $2.5 \%$ glutaraldehyde, washed in sodium cacodylate buffer, post-fixed in $2 \%$ osmium tetroxide, washed again in the same buffer, dehydrated in serial-graded acetone batches, impregnated and embedded in epon resin. The blocks were cut into $0.5-\mu \mathrm{m}$-thick sections (Ultramicrotome Reich, Germany) and stained with toluidine blue.

Only 83 of the 144 PNL samples and 187 of the 196 NLN samples had histopathological results for semi-thin sections available; therefore, this method was not employed for all the samples.

All sections were examined with a Nikon Eclipse E400 optical microscope (Nikon, Japan). Semi-thin sections allow for a more accurate quantification of nerve fibres and are appropriate for detecting morphological nerve fibre alterations, such as remyelination and axonal degeneration that are not clearly visible in paraffin sections.

Each histopathological alteration in the sample sections was labelled or not, according to the results of optical microscopy performed by two pathologists with leprosy experience. The frequency of each histopathological 
alteration in the PNL and NLN groups was compared with the chi-squared test according to Pearson or Fisher (2-tailed analyses) whenever correction was necessary.

The analyses emphasised determining the frequency of these alterations in AFB-negative PNL samples and comparing the AFB-negative PNL and NLN groups to determine a probable PNL diagnosis in the absence of AFB. One unequivocal indication of leprosy disease is the presence of AFB. In its absence, however, it is necessary to consider the possibility that nonspecific alterations may be signs of the disease because they are also detected in neuropathies other than leprosy. This work attempts to show that the presence of certain nonspecific histological alterations, such as fibrosis, subperineurial oedema and perineurial enlargement, are more frequent in leprosy than in NLN.

\section{RESULTS}

Overview of the histopathological alterations found in biopsy specimens - The presence of AFB in either Schwann cells or macrophages in the nerve, whether associated with inflammatory infiltrate or not, was the one finding that unequivocally guaranteed a specific PNL diagnosis. This evidence was detected in 35 PNL samples. We were aware that caution should be taken with myelin debris stained for AFB detection, which sometimes takes the shape of fuchsin-stained rods, misleading the observer searching for acid-fast structures. Other nonspecific alterations usually accompanied this main finding. Only eight AFB-positive samples lacked inflammatory infiltrate in the sections and the bacilli in the Schwann cell cytoplasm were in very small quantities. Among the 124 samples with M. leprae DNA confirmed by PCR, 28 of the 92 AFB-negative samples had a positive PCR test. The diagnosis of the remainder of the AFB-negative PNL samples relied on the presence of serum phenolic glycolipid antibodies (anti-PGL1) and the analysis of each patient's complete dataset.

Other histopathological alterations listed in this section are nonspecific and are found in other neuropathies. Most of these alterations appeared more frequently in PNL than NLN (Table II).

Generally speaking, the abnormal histological findings found in these groups of biopsy specimens were epineurial, perineurial and endoneurial inflammatory infiltrates, which are commonly composed of mononuclear infiltrate, epithelioid granuloma or foamy macrophages. Subperineurial oedema, a very early inflammatory sign, was also seen (Figs 1F, 2E). Fibrosis was found in the epineurium, perineurium and endoneurium (Figs 1B, D, 2B). Twenty-three samples in PNL group (21.1\%) displayed concomitant fibrosis of these three nerve compartments. Microfasciculation, a pattern first reported in leprosy by Vallat et al. (1991) and Antunes et al. (2011), was only observed in the PNL group. In addition, perineurial thickening (Figs 1E, 2B) was much more frequently observed within the PNL group, whereas Schwann cell proliferation was more commonly detected in the NLN group (see comparisons below).

Regarding the semi-thin sections, a reduced number of myelinated fibres, axonal remyelination (fibres with a lower relative myelin thickness) and axonal degeneration and regeneration were detected upon optical microscopy examination. This lower number of myelinated fibres

TABLE I

General data on nerve biopsy specimens and serum anti-persistent generalized lymphadenopathy (PGL)1

\begin{tabular}{lc}
\hline & $\mathrm{n}(\%)$ \\
\hline Pure neural leprosy (PNL) nerves & 144 \\
Acid-fast bacilli (AFB)-negative PNL nerves & $109(75.6)$ \\
AFB-positive PNL nerves & $35(24.3)$ \\
PNL nerves in which polymerase chain reaction (PCR) for Mycobacterium leprae DNA detection was done & 124 \\
M. leprae DNA-positive PNL samples by PCR & $53(42.7)$ \\
M. leprae DNA-negative PNL samples by PCR & $71(57.2)$ \\
PNL patients investigated for serum anti-PGL1 antibodies & 114 \\
Anti-PGL1-positive PNL patients & $54(47.3)$ \\
Anti-PGL1-negative PNL patients & $60(52.6)$ \\
PNL nerves with semi-thin histopathology available & 83 \\
Non-leprosy nerves (NLN) & 196 \\
NLN nerves in which PCR for M. leprae DNA detection was done & 150 \\
M. leprae DNA-positive PNL samples & 0 (0) \\
NLN patients investigated for serum anti-PGL1 antibodies & 93 \\
Anti-PGL1-positive NLN patients & $14(15)$ \\
Anti-PGL1-negative NLN patients & 79 (84.9) \\
NLN nerves with semi-thin histopathology available & 187 \\
\hline
\end{tabular}


and the other previously mentioned nerve fibre alterations were found, though much less efficiently, in sections stained with Gomori's trichrome.

Schwann cell proliferation was primarily detected as clusters of overlapping cell nuclei along the length of myelinated nerve fibres in haematoxylin-eosin-stained longitudinal sections or as an onion-bulb formation in transverse semi-thin sections.

Because leprosy is an infectious and inflammatory neuropathy, the PNL specimens displayed inflammatory infiltrate at a significantly higher rate than the NLN specimens. As expected, the cellular composition of

TABLE II

Frequencies of histopathological alterations

\begin{tabular}{|c|c|c|c|}
\hline & $\mathrm{n}(\%)$ & $\mathrm{n}(\%)$ & $\mathrm{p}$ \\
\hline & $\begin{array}{c}\mathrm{AFB}+\mathrm{PNL} \\
(\mathrm{n}=35)\end{array}$ & $\begin{array}{c}\text { AFB - PNL } \\
(\mathrm{n}=109)\end{array}$ & \\
\hline Epineurial infiltrate & $17(48.5)$ & $22(20.3)$ & $<0.00113$ \\
\hline Perineurial infiltrate & $25(73.5)$ & $31(28.7)$ & $<0.00001$ \\
\hline Endoneurial infiltrate & $27(77.1)$ & $29(27.1)$ & $<0.00001$ \\
\hline Mononuclear infiltrate & $25(73.5)$ & $35(32.1)$ & $<0.00002$ \\
\hline Epithelioid granuloma & $5(14.7)$ & $14(12.9)$ & $<0.77738$ \\
\hline Foamy macrophages & $19(55.8)$ & $7(6.5)$ & $<0.00001$ \\
\hline Epineurial fibrosis & $10(28.5)$ & $28(25.9)$ & $<0.75815$ \\
\hline Perineurial fibrosis & $13(37.1)$ & $37(34.2)$ & $<0.75588$ \\
\hline Endoneurial fibrosis & $12(34.2)$ & $34(31.4)$ & $<0.75759$ \\
\hline Reduced number of myelin at fibre & $27(77.1)$ & $51(48.1)$ & $<0.00274$ \\
\hline Endoneurial oedema & $3(15)$ & $4(4.9)$ & $<0.13742$ \\
\hline Schwann cell proliferation & $0(0)$ & $1(0.9)$ & $<1$ \\
\hline \multirow[t]{2}{*}{ Perineurial enlargement } & $10(30.3)$ & $17(15.7)$ & $<0.06279$ \\
\hline & $\begin{array}{c}\text { PNL } \\
(\mathrm{n}=144)\end{array}$ & $\begin{array}{c}\text { NLN } \\
(\mathrm{n}=196)\end{array}$ & \\
\hline Epineurial infiltrate & $40(27.7)$ & $6(3)$ & $<0.00001$ \\
\hline Perineurial infiltrate & $57(39.8)$ & $1(0.5)$ & $<0.00001$ \\
\hline Endoneurial infiltrate & $57(39.8)$ & $4(2)$ & $<0.00001$ \\
\hline Mononuclear infiltrate & $61(42.3)$ & $11(5.6)$ & $<0.00001$ \\
\hline Epithelioid granuloma & $19(13.2)$ & $0(0.0)$ & $<0.00001$ \\
\hline Foamy macrophages & $27(19)$ & $1(0.5)$ & $<0.00001$ \\
\hline Epineurial fibrosis & $38(26.3)$ & $8(4)$ & $<0.00001$ \\
\hline Perineurial fibrosis & $50(34.7)$ & $11(5.6)$ & $<0.00001$ \\
\hline Endoneurial fibrosis & $46(31.9)$ & $8(4.1)$ & $<0.00001$ \\
\hline Reduced number of myelin at fibre & $79(55.6)$ & $67(34.1)$ & $<0.00009$ \\
\hline Endoneurial oedema & $8(7.8)$ & $2(1.4)$ & $<0.01974$ \\
\hline Schwann cell proliferation & $1(0.7)$ & $37(19)$ & $<0.00009$ \\
\hline \multirow[t]{2}{*}{ Perineurial enlargement } & $27(19)$ & $14(7.1)$ & $<0.00097$ \\
\hline & $\begin{array}{c}\text { AFB - PNL } \\
(\mathrm{n}=109)\end{array}$ & $\begin{array}{c}\text { NLN } \\
(\mathrm{n}=196)\end{array}$ & \\
\hline Epineurial infiltrate & $22(20.3)$ & $6(3)$ & $<0.00001$ \\
\hline Perineurial infiltrate & $31(28.7)$ & $1(0.5)$ & $<0.00001$ \\
\hline Endoneurial infiltrate & $29(27.1)$ & $4(2)$ & $<0.00001$ \\
\hline Mononuclear infiltrate & $35(32.1)$ & $11(5.6)$ & $<0.00001$ \\
\hline Epithelioid granuloma & $14(12.9)$ & $0(0)$ & $<0.00001$ \\
\hline Foamy macrophages & $7(6.5)$ & $1(0.5)$ & $<0.00001$ \\
\hline Caseous necrosis & $3(2)$ & $0(0)$ & $<0.10095$ \\
\hline Epineurial fibrosis & $28(25.9)$ & $8(4)$ & $<0.00001$ \\
\hline Perineurial fibrosis & $37(34.2)$ & $11(5.6)$ & $<0.00001$ \\
\hline Endoneurial fibrosis & $34(31.4)$ & $8(4.1)$ & $<0.00001$ \\
\hline Reduced number of myelin at fibre & $51(48.1)$ & $67(34.1)$ & $<0.01789$ \\
\hline Endoneurial oedema & $4(4.9)$ & $2(1.4)$ & $<0.19606$ \\
\hline Schwann cell proliferation & $1(0.9)$ & $37(19)$ & $<0.00001$ \\
\hline Perineurial enlargement & $17(15.7)$ & $14(7.1)$ & $<0.01775$ \\
\hline
\end{tabular}

AFB: acid-fast bacilli; NLN: non-leprosy peripheral neuropathy; PNL: pure neural leprosy. 
the infiltrate consisted of mononuclear infiltrates with macrophages turning into either epithelioid cells (tuberculoid lesions) or AFB-loaded foamy macrophages (lepromatous lesions). Nevertheless, there were 22 samples $(15.2 \%)$ in which macrophages did not differentiate to either pole. Instead, they retained their mononuclear appearance and contained reniform nuclei in the peripheral cytoplasm and an ill-defined cellular border (Fig. 1A, B). Moreover, the concomitant presence of epithelioid granulomas and AFB-loaded vacuolated macrophages in the same nerve was found in five PNL specimens (3.4\%).

The reactive morphological alterations of inflammation, such as fibrosis, subperineurial oedema and perineurial thickening, were found in both groups; however, they were more frequent in PNL specimens. Fibrosis was identified as an enlarged, densely green area encompassing fibroblastic and inflammatory cells in transverse or longitudinal sections stained with Gomori's trichrome. The accumulation of green matrix among increased numbers of perineurial layers was considered to be a sign of perineurial fibrosis (Fig. 1E). Perineurial thickening was evidenced by a growing number of perineurial layers. Subperineurial oedema, a pre-inflammatory alteration, was shown by an enlargement of the subperineurial space containing microfibrillar material and sparse, non-clustered mononuclear cells (macrophages and/or lymphocytes).

Microfasciculation was discerned in 15 PLN samples (Fig. 2C, D). Microfascicles are nest-like structures surrounded by perineurial cells composed of a variable quantity of either small myelinated or non-myelinated fibres and by denervated Schwann cells and collagen fibres (Antunes et al. 2011).
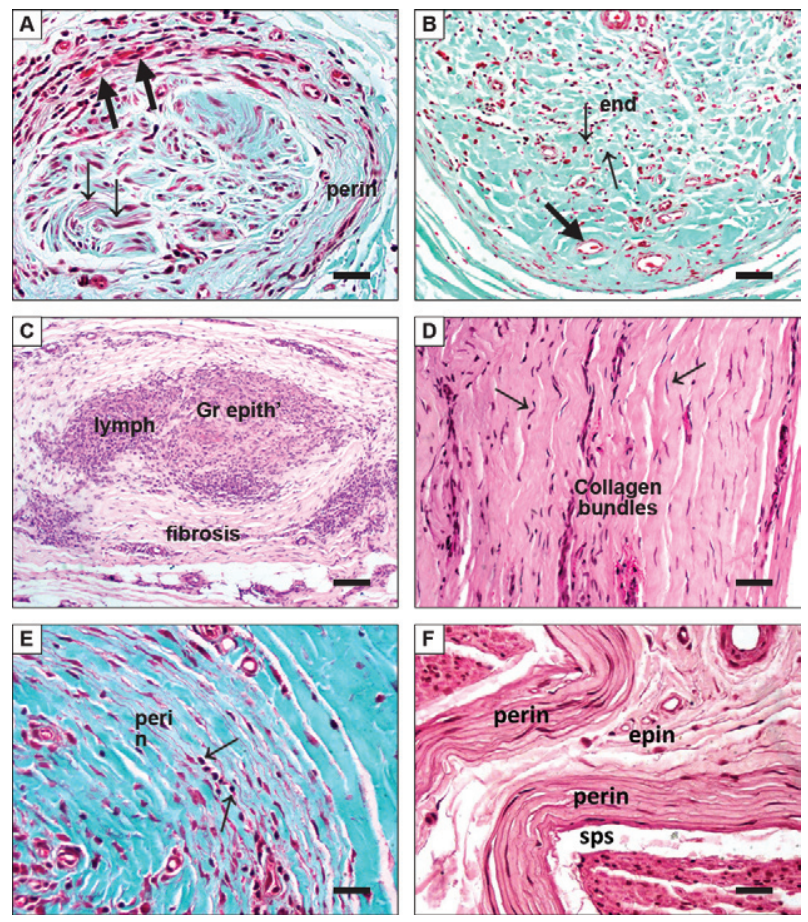

Fig. 1A: one nerve fascicle showing perineurial enlargement (perin) and mononuclear inflammatory infiltrate partially surrounding perineurial microvessels (arrows). Small myelinated fibres (thin arrows) can be seen in the endoneurium (end) compartment. Gomori's trichrome. Bar $=40 \mu \mathrm{m}$; B: one nerve fascicle showing sparse mononuclear cells (thin arrows) spread throughout the endoneurium. Absence of myelinated fibres and presence of angiogenic vessels with thickened walls (thick arrows). Gomori's trichrome. Bar $=40 \mu \mathrm{m}$; C: one nerve fascicle showing an epithelioid granuloma (gr epith) surrounded by a collar of lymphocytes (lymph). Endoneurial fibrosis erasing the perineurial, endoneurial and epineurial boundaries can also be seen. Haematoxylin-eosin (H\&E) staining. Bar $=80 \mu \mathrm{m}$; D: one nerve fascicle showing longitudinallyarranged parallel bundles of collagen fibres aligned with endoneurial fibroblasts (arrows) characterizing endoneurial fibrosis. H\&E staining. Bar $=20 \mu \mathrm{m}$; $\mathrm{E}$ : one nerve fascicle showing perineurium and mononuclear inflammatory infiltrate among the perineurial layers (arrows). Gomori's trichrome. Bar $=20 \mu \mathrm{m}$; F: partial view of two nerve fascicles and intervening epineurium (epin) showing thickened perineurium and enlargement of subperineurial space (sps) with irregular microfibrillar material within. H\&E. Bar $=20 \mu \mathrm{m}$.
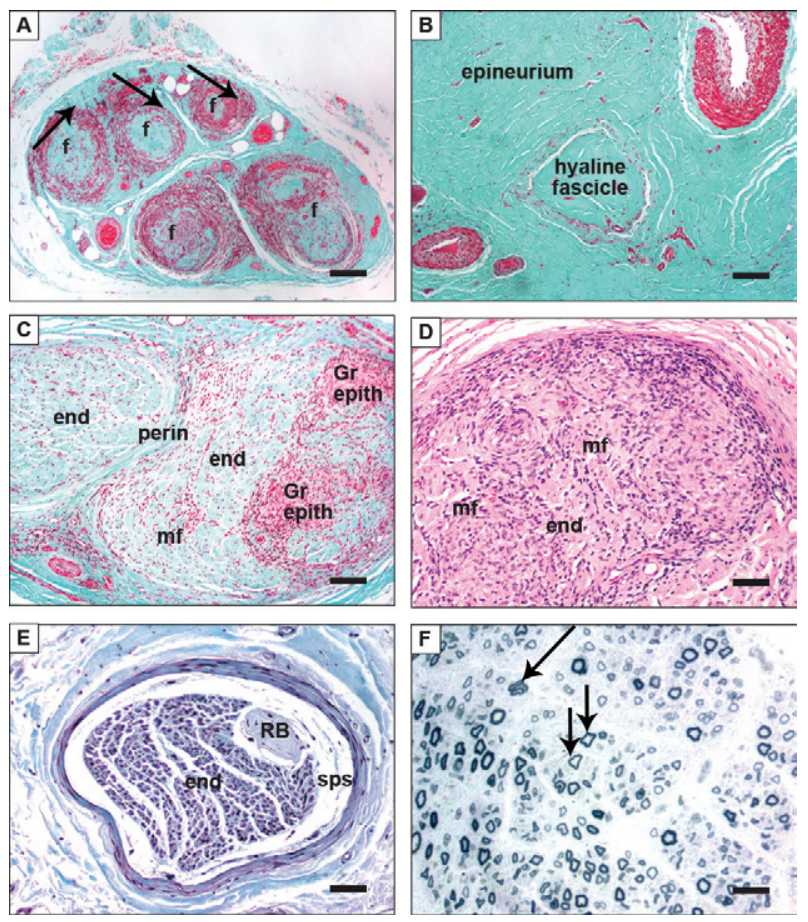

Fig. 2A: cross section of a peripheral nerve showing five fascicles (f) with mononuclear inflammatory infiltrate surrounding a fibrotic endoneurial compartment (arrows) devoid of nerve fibres. Gomori's trichrome. Bar $=200 \mu \mathrm{m}$; B: a fibrotic nerve fascicle showing a hyaline aspect and no fibre. The epineurium is also densely fibrotic. Gomori's trichrome. Bar $=80 \mu \mathrm{m}$; C: two nerve fascicles divided by a perineurial septa (perin) show the absence of myelinated nerve fibres and an irregularly spreading focus of epithelioid granulomas ( $\mathrm{gr}$ epith) in the endoneurium (end). Microfasciculation (mf) can be seen in the lower left quadrant of the same fascicle. Gomori's trichrome. Bar $=80 \mu \mathrm{m}$; $\mathrm{D}$ : one nerve fascicle showing $\mathrm{mf}$ in the end. $\mathrm{mf}$ are nest-like, round structures surrounded by perineurial cells containing nuclei and pink-stained material. Haematoxylin-eosin staining. Bar = $40 \mu \mathrm{m}$; E: one nerve fascicle showing enlargement of subperineurial space (sps) and microfibrillar material within. A Renaut body (RB) can be seen in the endoneurium. Gomori's trichrome. Bar $=40 \mu \mathrm{m}$; F: partial view of endoneurium showing a reduced number of large and small myelinated fibres, sprouting regenerating nerve fibres (long arrows) and few remyelinated fibres with a relatively thin myelin sheath (short arrows). Toluidine-blue staining. Bar $=20 \mu \mathrm{m}$. 
Regarding myelinated fibres, both Gomori's trichrome-stained and toluidine blue-stained semi-thin sections revealed a higher frequency of slightly to severely reduced numbers of myelinated fibres. Among the 83 biopsy specimens examined with semi-thin histology, 78 exhibited fibre loss, in contrast with 58 of the Gomori-stained paraffin sections. Unmyelinated fibres were not considered in this study because they can only be seen with ultrathin sectioning and transmission electron microscopy.

Remyelination, axonal degeneration and regeneration were detected by the presence of fibres with a relatively thin myelin sheath, which occurred less often in the PNL group and will be discussed in the following sections. Seven samples from the PNL group displayed no histopathological changes in either the paraffin or semi-thin histopathological examinations.

Comparing histopathological alteration frequencies - To assess the diagnostic value of each histopathological alteration found in the AFB-negative biopsy specimens, we compared their frequencies in the selected groups and subgroups (Tables II, III).

Multiple logistic regressions were used to identify variables that were independently associated with each group (Table IV). In the comparison between AFBnegative and AFB-positive PNL samples, perineurial enlargement remained statistically significant. This variable was able to correctly classify $69.7 \%$ of the AFB-positive PNL samples and $15.7 \%$ of the AFB-negative PNL samples.

In comparing the PNL and NLN samples, endoneurial infiltrate, perineurial fibrosis and perineurial enlargement also remained statistically significant. Collectively, these three variables correctly assigned $94.3 \%$ of the PNL samples and $6.2 \%$ of the NLN samples.

Mononuclear infiltrate and perineurial fibrosis also remained statistically significant in the comparison of AFB-negative PNL and NLN samples. Together, mononuclear infiltrate and perineurial fibrosis correctly detected $100 \%$ of the AFB-negative PNL samples and $0 \%$ of the NLN samples.

The data regarding the conclusions obtained from the histological examination of PNL nerve samples were divided into the following categories: (i) confirmed diagnosis [35 samples (24.3\%): inflammatory infiltrate composed of AFB-loaded macrophages or Schwann cells distributed across nerve compartments], (ii) highly probable diagnosis [14 samples $(12.9 \%)$ : the presence of epithelioid granulomas in the absence of AFB], (iii) probable diagnosis [2 samples $(15.2 \%)$ : the presence of inflammatory infiltrate composed exclusively of mononuclear cells without transformation into either epithelioid cells or AFB-loaded macrophages in the nerve compartments, particularly in the endoneurium and in the absence of AFB], (iv) possible diagnosis [66 samples (45.8\%): noninflammatory histological alterations were the only findings in the sample examination; these alterations occurred more frequently in PNL than NLN] and (v) nerves with normal histological appearance [7 samples (5.1\%)].
TABLE III

Comparison of restricted groups

Samples showing exclusively mononuclear infiltrate (PNL x NLN)

\begin{tabular}{lccc}
\hline & $\begin{array}{c}\text { PNL } \\
(\mathrm{n}=22) \\
\mathrm{n}(\%)\end{array}$ & $\begin{array}{c}\mathrm{NLN} \\
(\mathrm{n}=10)\end{array}$ & \\
$\mathrm{n}(\%)$ & $\mathrm{p}$ \\
\hline Perineurial fibrosis & $16(72.7)$ & $2(20)$ & $<0.00838$ \\
Endoneurial fibrosis & $14(63.6)$ & $2(20)$ & $<0.02212$ \\
Schwann cell proliferation & $0(0)$ & $3(30)$ & $<0.02419$ \\
\hline
\end{tabular}

Inflammation-free samples

\begin{tabular}{lccc}
\hline & $\begin{array}{c}\text { PNL } \\
(\mathrm{n}=66) \\
\mathrm{n}(\%)\end{array}$ & $\begin{array}{c}\mathrm{NLN} \\
(\mathrm{n}=187) \\
\mathrm{n}(\%)\end{array}$ & $\mathrm{p}$ \\
\hline Epineurial fibrosis & $11(13.2)$ & $6(3.2)$ & $<0.00172$ \\
Perineurial fibrosis & $13(15.6)$ & $9(4.8)$ & $<0.00264$ \\
Endoneurial fibrosis & $8(9.6)$ & $6(3.2)$ & $<0.02959$ \\
Schwann cell proliferation & $1(1.2)$ & $35(18.9)$ & $<0.00010$ \\
\hline
\end{tabular}

Comparison of PNL $(\mathrm{n}=83) \times$ NLN $(\mathrm{n}=187)$ [semi-thin sections (st)]

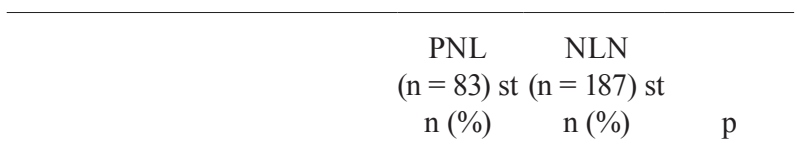

Loss of large myelinated fibres 78 (77.2) $110(64.3)<0.02609$

Loss of small myelinated fibres $77(77) \quad 113(66.4)<0.06729$

Signs of remyelination $\quad 11(11.3) \quad 53(32.5)<0.00013$

Signs of axonal degeneration $14(14.4) \quad 30(18.8)<0.36155$

Signs of axonal regeneration $\quad 11(11.1) \quad 37(23.1)<0.01561$

NLN: non-leprosy peripheral neuropathy; PNL: pure neural leprosy.

TABLE IV

Multivariate analysis Comparison of AFB + PNL x AFB - PNL

\begin{tabular}{lcc}
\hline & OR & $\mathrm{p}$ \\
\hline \multicolumn{1}{c}{ Comparison of PNL x NLN } & \\
Endoneurial infiltrate & 2.5 & $<0.001$ \\
Perineurial fibrosis & 2.8 & $<0.001$ \\
Perineurial enlargement & 1.8 & 0.006 \\
\multicolumn{1}{c}{ Comparison of AFB - PNL x NLN } & \\
Mononuclear infiltrate & 0.5 & 0.006 \\
Perineurial fibrosis & 1.8 & 0.002 \\
& 1.2 & $<0.001$ \\
\hline
\end{tabular}

AFB: acid-fast bacilli; NLN: non-leprosy peripheral neuropathy; OR: odds ratio; PNL: pure neural leprosy. 


\section{DISCUSSION}

The present study is a report of our hands-on experience with histopathologically diagnosing PNL. Diagnosis was based on nerve sample examination in the context of clinical, electroneuromyographical and laboratory data collected by an experienced medical team actively engaged in the diagnosis and health assistance of leprosy patients.

Important research in the literature demonstrates the relevance of nerve biopsy examinations to diagnosing leprosy neuropathy. This study also testifies to the first-hand experience of a leprosy reference centre under the auspices of the Brazilian Ministry of Health and how information extracted from the histopathological examination of PNL and NLN nerve samples can be effectively used to reach an accurate leprosy diagnosis. The value of each alteration, particularly those also present in nonleprosy neuropathies, was assessed by statistically comparing the alteration's frequency in the PNL and NLN groups.

Again, the classical studies on this subject published by Job (1989), Antia et al. (1970) and Dastur et al. (1970) have strong a descriptive value; they emphasise items that should be prioritised during the histopathological examination of nerve samples from suspected leprosy cases. Chimelli et al. (1997) and Garbino et al. (2004), in studies more closely related to the practical diagnostic importance of leprosy neuropathy, describe alterations that can be found in nerve biopsies in general.

The present study goes beyond the most characteristic histopathological alterations commonly associated with leprosy nerves while emphasising the morphological changes in AFB-negative leprosy nerve specimens. In addition, this study compared the frequency of each alteration in PNL and NLN nerve samples to determine the value of each one when making a PNL diagnosis.

As previously described, the conclusions related to histopathological alterations were categorised into five groups. In our view, the first category justifies diagnosing leprosy disease based on the biopsy alone, in which case the presence of AFB may or not be accompanied by a variety of inflammatory and structural alterations in the nerve.

Problems arise when AFB is not detected in the sample. In this circumstance, the presence of epithelioid granuloma in the nerve signals a high probability of leprosy, particularly when the granuloma is in the endoneurial compartment. Another rare aetiology in this case could be neural sarcoidosis. Said et al. (2002) reported 11 cases and granulomas were restricted to the epineurium in all of them. However, no diagnosis of sarcoidosis was confirmed in the present study.

The concomitant presence of epithelioid granulomas and AFB-loaded foamy macrophages also indicates the presence of opposite extremes of the immunoinflammatory response (Thelperl and Thelper2, which, respectively, correspond to the paucibacillary and multibacillary nerves) (Modlin 1994) in the same PNL biopsy specimen. This concomitant presence could identify an episode of reactional neuritis (intraneural reactions without cutaneous lesions) or perhaps a borderline type of infiltrate instead. Even so, the definition of a reactional diagnosis depends first and foremost on the existence of neuritic pain.
Regarding the third category, which includes samples that show an exclusively mononuclear cell infiltrate in the absence of granulomas or foamy macrophages, it is worth remarking that other inflammatory disorders of the peripheral nerves, such as vasculitic neuropathy and chronic inflammatory demyelinating polyneuropathy, could also present this pattern and should be kept in mind as differential diagnoses.

Increased perineurial layers, assumed to be a nonspecific finding, are significantly more frequent in the PNL group. CD34-immunolabelling revealed that perineurial thickening might be due to the differentiation and apposition of epineurial fibroblasts to the external layers of the perineurium (unpublished observations). The role of the perineurium in leprosy-related nerve damage remains to be clarified; nonetheless, these special cells are likely involved, as they are a part of the blood-nerve barrier and adjacent to the traversing perineurial vessels from which the leprosy inflammatory infiltrate most often emerges.

In almost all comparisons performed in the present study, fibrosis was the most consistent non-inflammatory alteration in the PNL group as a whole (i.e., both the AFBpositive and negative specimens). It is known that leprosy leads to characteristically dense fibrosis during the end stages of nerve damage (Junqueira et al. 1980, Singh et al. 1998). Therefore, it would seem plausible to assert that fibrosis in the nerve should, at the very least, raise the suspicion of leprosy. Moreover, because subperineurial oedema can be viewed as a sign of fluid exchange imbalance within the nerve, as well as a possible reflex of an early inflammatory process, this finding should likewise alert clinicians to the possibility of a leprosy diagnosis.

In addition, multivariate analysis showed that special attention should be paid to perineurial fibrosis, perineurial enlargement, mononuclear infiltrate and endoneurial infiltrate, as they were independently more frequent in the PNL group and, when detected together, could identify PNL in $100 \%$ of AFB-negative samples.

A reduction in the number of myelinated fibres assessed with Gomori's trichrome and toluidine bluestained semi-thin sections was probably due to the greater destructive power of the inflammatory infiltrate present in the PNL samples. One finding corroborating this hypothesis is that this significant difference disappeared when inflammation-free samples from both groups were compared.

The chromotrope 2R used in Gomori's trichrome stains myelin red and allows for a gross quantitative assessment of these fibres and the easy detection of myelin ovoids. The routine processes of formaldehyde fixation, alcohol dehydration, xylene clarification and paraffin embedding lead to profound alterations in myelin structure and hamper the analysis of fine damage to the myelinated nerve fibres. The examination of semi-thin sections could be considered a more sensitive method for detecting quantitative and qualitative alterations in myelinated fibres.

Semi-thin section histology did not directly increase the sensitivity of the PNL diagnosis. However, it did improve the visualisation of some non-inflammatory findings, such as perineurial oedema and perineurial 
thickening. It also led to the diagnosis of other peripheral neuropathies by revealing fine alterations in the nerve fibres that were not observable in paraffin-embedded sections.

The lower rate of remyelination and active axonal degeneration in the PNL group prompts a controversial discussion about the mechanisms involved in nerve fibre damage in leprosy neuropathy, a subject beyond the scope of this study.

The selection of a nerve trunk for biopsy is based on evidence of clinical alteration in the nerve territory and motor conduction or sensitivity disturbances in the trunk. The consistently uneven damage patterns of leprosy-affected nerves may explain why seven PNL samples had a normal morphological appearance despite abnormal tests. It is conceivable that a normal segment might have been collected during the biopsy procedure, making a focal or more proximal lesion undetectable. In these cases, the PNL diagnosis was based on the detection of $M$. leprae DNA in the nerves and/or anti-PGL1 antibodies in patient's sera.

In summary, the present study attempted to maximise the amount of information obtained from the histopathological examination of nerve samples from suspected PNL patients. We found higher frequencies of some noninflammatory histopathological alterations in the PNL samples, which could be considered strong indicators of a leprosy diagnosis regardless of AFB negativity.

\section{ACKNOWLEDGEMENTS}

To Judy Grevan, for editing the text.

\section{REFERENCES}

Antia NH, Pandya SS, Dastur DK 1970. Nerves in the arm in leprosy. 1. Clinical, electrodiagnostic and operative aspects. Int J Lepr Other Mycobact Dis 38: 12-29.

Antunes SL, Medeiros MF, Corte-Real S, Jardim MR, Amadeu TP, Nery JA, Hacker MA, Valentim VC, Sarno EN 2011. Microfasciculation: a morphological pattern in leprosy nerve damage. Histopathology 58: 304-311.

Chimelli L, Freitas M, Nascimento O 1997. Value of nerve biopsy in the diagnosis and follow-up of leprosy: the role of vascular lesions and usefulness of nerve studies in the detection of persistent bacilli. J Neurol 244: 318-323.
Dastur DK, Pandya SS, Antia NH 1970. Nerves in the arm in leprosy 2. Pathology, pathogenesis and clinical correlations. Int $J$ Lepr Other Mycobac Dis 38: 30-48.

Garbino JA, Ura S, Belone AF, Marciano LH, Fleury RN 2004. Clinical and diagnostic aspects of the primarily neural leprosy. Hansen Int 29: 124-129.

Girdhar BK 1996. Neuritic Leprosy. Indian J Lepr 68: 35-42.

Jardim MR, Antunes SL, Santos AR, Nascimento OJ, Nery JA, Sales AM, Illarramendi X, Duppre N, Chimelli L, Sampaio EP, Sarno EP 2003. Criteria for diagnosis of pure neural leprosy. J Neurol 250: 806-809.

Jardim MR, Antunes SL, Simons B, Wildenbeest JG, Nery JA, Illarramendi X, Moraes MO, Martinez AN, Oskam L, Faber WR, Sarno EN, Sampaio EP, Bührer-Sékula S 2005. Role of PGL-I antibody detection in the diagnosis of pure neural leprosy. Lepr Rev 76: 232-240.

Job CK 1989. Nerve damage in leprosy. Int J Lepr Other Mycobact Dis 57: 532-539.

Junqueira LC, Montes GS, Neto EA, Barros C, Tedesco-Marchese AJ 1980. The collagen of permanently damaged nerves in human leprosy. Int J Lepr Other Mycobact Dis 48: 291-297.

Modlin RL 1994. Th1-Th2 paradigm: insights from leprosy. J Invest Dermatol 102: 828-832.

Pannikar VK, Arunthathi S, Chacko CJ, Fritschi EP 1983. A clinicopathological study of primary neuriticleprosy. Lepr India 55: 212-221.

Said G, Lacroix C, Planté-Bordeneuve V, Le Page L, Pico F, Presles O, Senant J, Remy P, Rondepierre P, Mallecourt J 2002. Nerve granulomas and vasculitis in sarcoid peripheral neuropathy: a clinicopathological study of 11 patients. Brain 125: 264-275.

Shetty VP, Antia NH 1997. Pathology of nerve damage in leprosy. In The peripheral nerve in leprosy and other neuropathies, Oxford Press, Calcuta, p. 79-137.

Singh N, Birdi TJ, Chandrashekar S, Antia NH 1998. In vitro studies on extracellular matrix production by $M$. leprae infected murine neurofibroblasts. Lepr Rev 69: 246-256.

Srinivasan H 1990. Nerve damage, surgery and rehabilitation in leprosy. Trop Med Parasitol 41: 347-349.

Vallat JM, Leboutet MJ, Henry P, Millan J, Dumas M 1991. Endoneurial proliferation of perineurial cells in leprosy. Acta Neuropathol (Berl) 81: 336-338.

WHO - World Health Organization 2010. Global leprosy situation 2010. Wkly Epidemio Rec 85: 337-334. 